\title{
Cyber Physical Socio Ecology
}

\author{
Xiaoqing $\mathrm{Shi}^{1}$ and Hai Zhuge $\mathrm{e}^{2,3, *, \dagger}$ \\ ${ }^{1}$ State Key Laboratory of Urban and Regional Ecology, Research Center for Eco-Environmental Sciences, \\ Chinese Academy of Sciences, Beijing, People's Republic of China \\ ${ }^{2}$ Institute of Computing Technology, Chinese Academy of Sciences, Beijing, People's Republic of China \\ ${ }^{3}$ Southwest University, People's Republic of China
}

\begin{abstract}
SUMMARY
Exploring the laws of the nature and the rules of human society is the grand challenge of sciences. The Internet, Web, various communication networks and digital devices are connecting each other to form an enormous cyber space. The cyber space gradually connects the natural physical space via various cyberphysical interfaces to form Cyber Physical Systems. Interacting with humans, Cyber Physical Systems will connect human society to form a new world-Cyber Physical Society. Humans' daily life and work will become increasingly relying on the new world. Billions of years' evolution creates the most elegant and efficient natural ecosystem. It will be ideal if the Cyber Physical Society can be an ecosystem that harmoniously evolves with society, economy, culture, sciences and technologies. The natural ecosystem gives us inspiration to ensure the harmony of the Cyber Physical Society. From ecosystem point of view, this paper proposes the notion, method, scientific issues, general architecture and resource management of Cyber Physical Society. The study of the Cyber Physical Socio Ecology (CPSE) could lead to a new branch of sciences. Copyright (C) 2010 John Wiley \& Sons, Ltd.
\end{abstract}

Received 4 May 2010; Accepted 16 May 2010

KEY WORDS: Cyber Physical System; future interconnection environment; digital ecosystem

\section{INTRODUCTION}

Computers have evolved from mainframes to networked desktops, and then to mobile and ubiquitous computing via the Internet, Web and wireless networks. Various networks and devices will be connected to form a global cyber space. It will gradually connect the natural space via various cyber physical interfaces like sensors to form a global cyber physical system.

A cyber physical system tightly coordinates its computational elements and physical elements rather than only computational elements as previous computer systems. It has recently become high priority in NSF research agenda. The vision of cyber-infrastructure for 21 st century discovery was proposed by NSF of U.S.A. in 2006.

Much effort from different aspects has been made to improve the cyber space [1-7]. The cyber space will also connect the society via various devices related to humans to form a new

\footnotetext{
*Correspondence to: Hai Zhuge, Institute of Computing Technology, Chinese Academy of Sciences, Beijing, People's Republic of China.

†E-mail: zhuge@ict.ac.cn

Contract/grant sponsor: International Cooperation Project of Ministry of Science and Technology of China; contract/grant number: 2006DFA11970

Contract/grant sponsor: National High Technology Research and Development Program of China; contract/grant number: 2007AA12Z220

Contract/grant sponsor: National Science Foundation of China; contract/grant numbers: 60773057, 60703018, 70773109
} 
world-Cyber Physical Society, which concerns not only the cyber space and the nature but also society, economy and culture.

The eco-Grid, proposed in 2004 from ecosystem point of view, is to create an environment that can harmoniously evolve with society, economy and technology [8]. Its architecture is proposed for managing various resources in this new world by balancing the service flow, knowledge flow, information flow and material flow according to social and economic principles. In 2005, a set of parameters of the future interconnection environment was proposed [9].

The Cyber Physical Society will also concern the development of knowledge and thought as they ultimately reflect the development of society [10]. The Knowledge Grid is an ideal intelligent and sustainable interconnection environment that enables people or roles to effectively capture, publish, share and manage knowledge resources. It provides appropriate services to support innovation, cooperative teamwork, problem-solving and decision making. It incorporates epistemology and ontology to reflect human cognition characteristics; exploits social, ecological and economic principles; and, adopts up-to-date networking techniques and standards [11, 12]. It will develop toward an intelligent interconnection environment that consists of autonomous individuals, self-organized semantic communities, an adaptive networking mechanism, an evolving semantic overlay keeping meaningful connection between individuals, flows for dynamic resource sharing and mechanisms for supporting effective resource management and providing appropriate knowledge services for problem-solving and innovation [9]. Its long-term target is to support innovation and harmonious development of science, technology and culture. It can be regarded as the high-level architecture of the Cyber Physical Society.

Social networks have drawn increasingly attention in many disciplines $[13,14]$, but current research on social networks mainly focuses on graph analysis. Social networks in real applications are usually involved in rich semantics. Effort to create interactive semantics has been made for establishing the understanding basis for the future interconnection environment $[15,16]$. Any individual and interaction will have semantic image in the future Cyber Physical Society [10].

This paper proposes the Cyber Physical Socio Ecology (CPSE) as the method of studying and developing the Cyber Physical Society. It includes fundamental concepts, models, methods, scientific issues and resource management.

\section{FROM NATURAL ECOSYSTEM TO CYBER PHYSICAL SOCIETY}

Through experienced billions of years' evolution, the huge and complex natural ecosystem has become the most elegant and efficient system. Ecology is a study of organism, natural environment and society [17]. It concerns the structure and function of ecosystem as well as the relationship between organisms and the environment.

Food web plays an important role in balancing the population of communities in the nature. Material flow works with the material web when materials pass through material transformation process. Nutrition flow works with the food web when nutrition passes through individuals. Energy flow is formed when it is transformed from one tropical level into another in the food web. Information flow goes through individuals within a community for inheritance and communication.

Figure 1 shows a simple natural ecosystem model with material flows. Some individuals play the roles of both consumer and producer. A healthy ecosystem works with effective energy flow, material flow and information flow cycles, by which the system can automatically keep dynamic balance on the population of all communities involved in the flow cycles, assimilate waste, selfrecover a certain degree of damage, and keep harmonious and sustainable evolution. The excellent characteristics of the natural ecological environment enlighten a new way to study and develop the Cyber Physical Society [8].

Extending cyber space to the nature will include the following parameters: space-the capacity to encompass a great variety of individuals, time - the arrow of evolution, structure - the construction of the environment and its individuals, relation - the relationships between parameters and between 


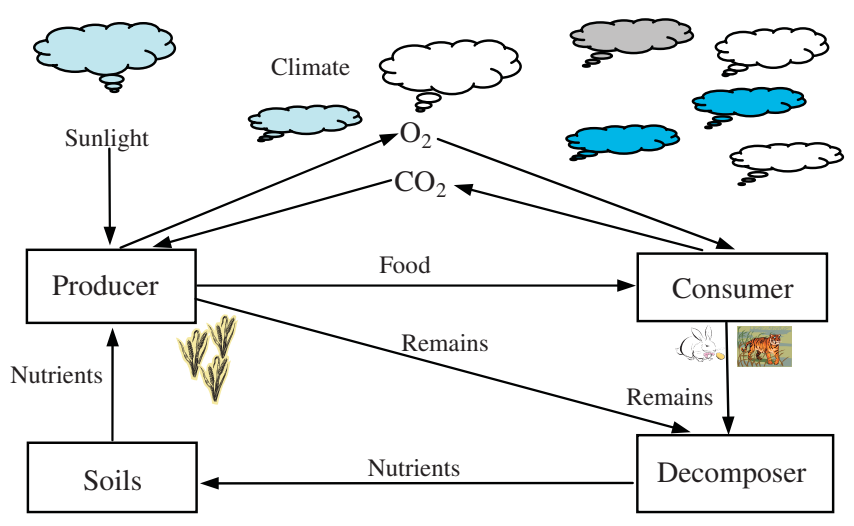

Figure 1. A simple natural ecosystem with material flows.

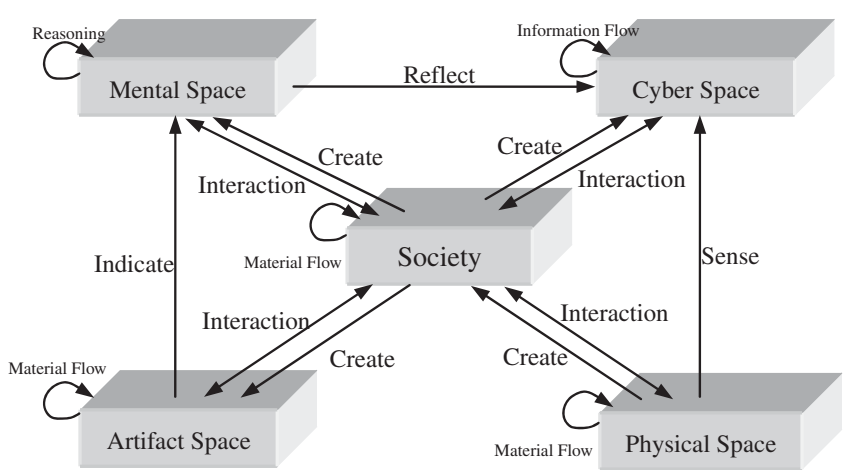

Figure 2. Interaction among spaces of the Cyber Physical Society.

individuals, and worth - the evaluation of the status of, and the prospects for, individuals, processes and their relationships [9].

The development, operation and maintenance of the environment will involve in the following spaces, which will interact with each other as shown in Figure 2:

(1) The natural physical space-the nature and natural materials.

(2) Artifact space-a space consists of artifacts such as buildings, material culture, devices and networks.

(3) The mental space-ideals, religions, morals, non-material culture, arts, wisdom, know-how, knowledge of sciences, springing from thought, emotion, creativity and imagination.

(4) The cyber space - the perceptual environment that supports vision, hearing and possibly touch. It is supported by various digital devices and networks. It will more and more reflect the physical space and mental space.

The society involves the above spaces. It consists of human individuals, relevant artifacts, and social regulations. Human bodies belong to the physical space, whereas their thoughts belong to the mental space, and the products of their thoughts belong to the physical space or the cyber space.

The society will develop through various interactions. The cyber space starts through interaction between the physical space and the society, and then evolves through interactions among these spaces. The cyber space directly influences the society and indirectly influences the physical space through the society and the physical space itself.

The cyber physical society will develop under the principles of openness, incremental development, economy, ecology, competition and cooperation, elasticity, integrity and uniformity. 


\section{DEFINITION, SCIENTIFIC ISSUES AND METHOD}

CPSE studies harmonious development of the spaces as an entire environment. It concerns the architecture and function of the environment, methods and technologies for developing the environment, and the intrinsic relationships and rules in the environment.

CPSE studies the following fundamental scientific issues:

(1) Individual model. Research should answer what is the individual in the Cyber Physical Society as well as its structure, function and generation mechanism. Individual organism is the most basic elements in the Cyber Physical Society.

(2) Species model. In the Cyber Physical Society, individuals that share the same features and often interact with each other tend to constitute species. To determine the number of individuals and their relations within a species as well as the diversity of species in society are important research issues.

(3) Relationship and interaction rules between individuals, and between species. Based on the individual model and species model, research should answer: What is the symbiosis relation between species? What is the nature of the interaction within the Cyber Physical Society? It should be different from the interaction in the current Web and also different from the direct conversation between people. At the low level, is it similar to the interaction between organisms or between animals? At the high level, is it similar to the art languages? Research needs to answer these questions.

(4) Information fusion on demand. How different types of information can be fused on demand to provide services through multiple channels. Studying cognition and psychology on human's information fusion can help in-depth understanding of information fusion on demand.

(5) Competition and cooperation principles in the Cyber Physical Society. Competition, symbiosis and cooperation exist among individuals and among communities in the same evolution process of the Cyber Physical Society. What are the principles of these behaviors? Research result will suggest appropriate rules for competition and cooperation in the Cyber Physical Society.

(6) Evolution and adaptation. Cyber Physical Society consists of versatile individuals; hence, the evolution of each individual contributes to the evolution of the whole Cyber Physical Society. How different species influence the whole Cyber Physical Society? The evolution of culture may affect biological evolution [18]. How different types of individuals influence each other?

(7) Model and principle of flows. Research concerns the models and principles of service flow, knowledge flow, information flow, energy flow and material flow. These separated flows will be coordinated in Cyber Physical Society. How these flows support efficient execution of Cyber Physical Society? Comparison between these flows and those in natural ecosystem will benefit research.

(8) Health of Cyber Physical Society. Research should provide the method for evaluating the status of the Cyber Physical Society, judge whether it evolves in a sustainable way and provide the way to improve its health status.

(9) Methodology for developing and studying CPSE. It suggests research method for developing, analyzing and maintaining the Cyber Physical Society. Incremental development is a feasible way toward the Cyber Physical Society. Comparison between the natural ecosystem and the Cyber Physical Society at every development stages will inspire research.

Philosophical issues will emerge when the Cyber Physical Society becomes an environment where people live and work with. This is because the reality will become Cyber Physical Society. Studying domain-specific Cyber Physical Society will help people understand and study its principles.

The natural ecosystem works with natural law. Natural ecology is the recognition of the natural ecosystem and the relationship between human society and natural ecosystem. Energy flow, information flow and material flow in natural ecosystem is governed by natural laws as shown in 


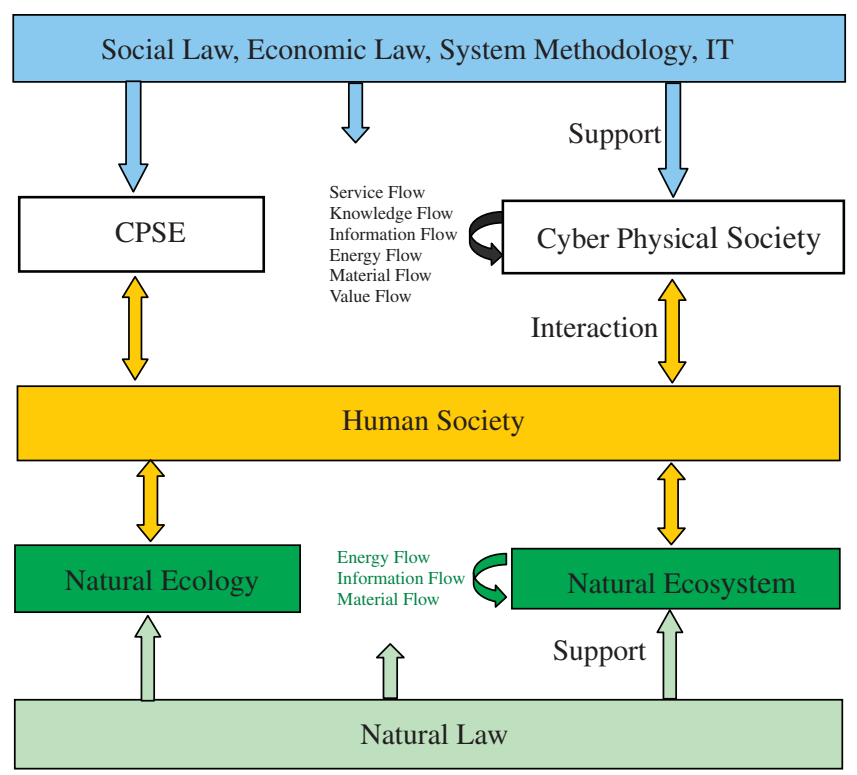

Figure 3. Relations among Cyber Physical Society, CPSE and natural ecology.

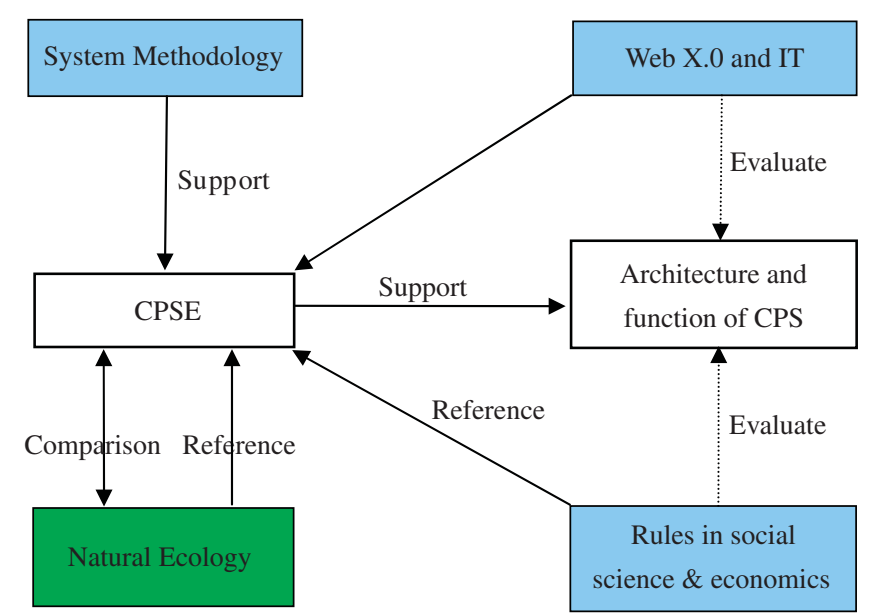

Figure 4. Research method of CPSE.

Figure 3. Corresponding principles, hypotheses and problems will be different in Cyber Physical Society.

Cyber Physical Society will execute according to social law, economic laws and information technology. Service flow, knowledge flow, information flow, energy flow, material flow and value flow implement the function of the Cyber Physical Society.

The cyber space facilitates the sharing of information. The food web guarantees the harmony of natural ecosystem. The Cyber Physical Society will facilitate the harmony of society.

Figure 4 suggests the general research method of CPSE. Since the Cyber Physical Society is a complex system, the system methodology can be used in general in CPSE research. Studying the rules of the hidden Web in society, economy and nature can help establish the fundamental rules of the Cyber Physical Society. Some principles in the natural ecosystem and society, such as the small-world [19], survival of fittest, the rich gets richer and the indirect reciprocity, can be used for reference in the Cyber Physical Society [20]. Rules in social science, management science and economics could also be used for reference in research of CPSE and in evaluation of research. 
The study of Web X.0 and the development experience of information theory and information technology (IT) will help choose the appropriate way to develop the Cyber Physical Society.

Establishing analogy among various flows in nature (e.g. energy flow and material flow), information flows in cyber space and the characteristics of the flows in Cyber Physical Society will benefit research on the principles of the flows in the Cyber Physical Society.

Hypotheses can be proposed by surveying the natural ecology and the rules in social science and economics. Since Cyber Physical Society is at very preliminary stage, scientific hypothesis and scenario analysis will be very important in CPSE research.

\section{INDIVIDUAL MODEL}

The world is constituted by versatile individuals and species. Just as the generation of life on the earth, the current passive Web pages and documents would evolve into live individuals in the future Cyber Physical Society.

Sixty years ago, Bush proposed the ideal of memex-a device that can store books, records and communications, and it can be mechanized to be consulted with exceeding speed and flexibility [21]. The invention of general-purpose computer and Internet largely realized the ideal.

What is the individual in the cyber space? Computer scientists have invented many individual models by abstraction. Object-oriented programming and development method use the notions of objects and classes to unify diverse abstractions and simplify the conceptualization of complex objective world [22]. Separating data from program is an important notion in computer science, which greatly promotes the study of data structure and algorithm. Agent and multi-agent technologies simulate individual and interaction between individuals [23].

Gray proposed the notion of personal memex and world memex [24]. The personal memex could record everything a person sees and hears, and quickly retrieve any item on request. The world memex could answer questions about the given text and summarize the text as precisely and quickly as a human expert in that field. Do the same for music, art and cinema.

A general individual model memex extension (ME) is configurable, adaptive and context-aware digital organism, modeling various types of network resource, hosting-distributed network software and device [25]. One ME can selectively inherit the function of another ME, and MEs can be composed into one with a richer function.

ME model will be the advanced development stage of information and knowledge. Information, knowledge and software can be unified within MEs. In macrocosm, MEs are self-organized by interacting with each other and with humans to hold an evolving ME society. A competition mechanism in this society can help improve its effectiveness. In microcosm, MEs are tightly coupled to perform the integrated software via information flow and internal control flow. Information and functions are encapsulated within MEs. In vision of ME, the behavior of software is the same as its structure. Sharing and dependence relations between resources establish the symbiosis relationship among MEs.

The ME model can use the following components to realize personal Web information service:

(1) Personalized crawler that collects resources satisfying personal interest from the Web.

(2) Evolving Resource Space that manages the collected resources by classification. Its structure keeps evolving with the increase of resources.

(3) Self-organized Semantic Link Network that self-organizes resources via various relations.

(4) Function set that includes such functions as discovering semantic communities, relational query, relational reasoning and maintenance operations.

(5) Detector that detects local capacities and other MEs on the Web for sharing and cooperation.

(6) Interface that interacts with humans or other MEs.

The future cyber space will be a world of MEs. With the increment of collected resources and interacting with people and with each other, an ME keeps evolving to provide better information 
service in efficiency and efficacy. The ME will also include cognitive robots. The resource sharing relations connect MEs into communities.

\section{FLOWS IN CYBER PHYSICAL SOCIETY}

Service flow, information flow, knowledge flow, value flow, energy and material flow connect individuals and organizations in different spaces of the Cyber Physical Society.

Service flows are at the high-level of the Cyber Physical Society. There are two motivations of generating the service flow: (1) the reliance between services and (2) the demand from social activities. Different from static services, service flow dynamically organizes services and provides appropriate service logistics for demanders according to the principles of management and economics. The study of workflow and service science will help this research [5].

Information transformation and transmission forms information flow. The study of information flow is to ensure efficient transmission of information through individuals and provision of necessary information for demanders. An effective information flow is relevant to users' interest and preference. Understanding Shannon's information theory can help study information flow [26].

Knowledge itself is power. Knowledge flow is the power of a team. The original force of knowledge flow comes from humans' problem-solving requirement, the intrinsic motivation of knowing the world and reputation. The study of knowledge flow is to guarantee efficient and effective knowledge sharing across spaces to promote the productivity of a virtual organization. An efficient and effective knowledge flow depends on the organization of individuals of different knowledge power, social principle and economic principle [27, 28].

Value flow is a product of social development, which does not exist in natural ecosystem. It accompanies exchange activities. It pursues the maximum growth when flowing through social activities. The value flow is accompanied with service flow, information flow, knowledge flow and material flow.

Material flow refers to the transmission of materials through individuals, organization, production processes and consumption processes in the Cyber Physical Society. Figure 5 depicts a material flow cycle from raw materials to products. Different stages concern different production processes

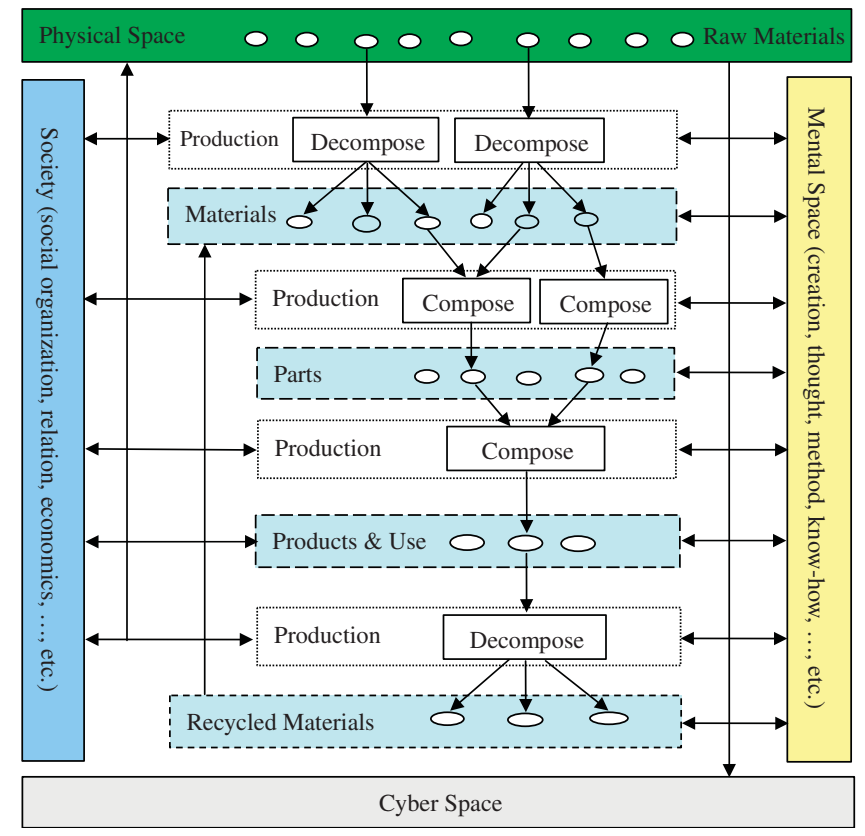

Figure 5. Material flow cycle in the Cyber Physical Society. 
and relevant social organization and innovation processes as well as different influences on social space and physical space. Decomposing end-of-use products is the key to realize green society.

Service flow, information flow, knowledge flow, value flow, energy flow and material flow often involve in the same process. Effective service flow needs material flow logistics and energy supply. Material flow will usually work with the value flow. How different flows influence each other? How they cooperate with each other to obtain efficiency and effectiveness? Research on CPSE needs to answer these questions.

Particular structure has been found in many complex networks, such as the Web, citation networks, email networks, food webs, social networks and biochemical networks: nodes are often clustered into tightly-knit groups, and edges are dense within groups and loose between groups $[13,14]$. Such a structure reflects the characteristic of human group behavior of sharing information $[2,29]$. Researches on discovering network communities have been done as graph partitioning in graph theory, computer science, hierarchical clustering in sociology and geographical partition. Previous community discovery approaches may not be suitable for semantics-rich network as implied semantic relations could be derived from existing relations, and adding a node or relation could influence existing nodes and relations and even the whole network [16]. Flows in Cyber Physical Society are dynamic and content relevant. Different types of flows follow different rules. Discovering communities in dynamic flows is an important issue to be studied.

\section{RELATION BETWEEN CYBER PHYSICAL SOCIETY AND OTHER SYSTEMS}

The Web 1.0 is mainly a read-only information sharing platform. Its characteristic is the easy usage mode and self-organization hyperlink architecture: one page can link to any other page. Society and culture have shown influence on forming the structure of the Web. The following phenomena can be observed: people tend to read web pages in the same language as they previously used, and a web page is likely to link to the page in the same language. These phenomena indicate that the Web is local to cultures and communities.

Web 2.0 provides a massively interactive information sharing platform. By this interactive feature, society and culture will influence the evolution of the Web more significantly. Web 2.0 has the ability of collective creation and annotation of various contents.

Web 1.0 uses the dominant technology: HTTP protocol, HTML-based web page design and the URL web page locating mechanism. Search engines are the complementary technology of Web 1.0. From Web 2.0, the Web will enter an era of diverse technologies and usage modes.

Web 3.0 is expected to be a semantics-rich Web based on both Web 1.0 and Web 2.0. Web 1.0 provides human understandable contents and Web 2.0 facilitates interaction. Research on Semantic Web, wisdom Web and Web of Things will converge into Web 3.0.

The evolution of software neglects the lower version. The new version completely covers the functions of old versions. Hence, people tend to pursue the new version. The characteristic of the Web evolution is that the old versions will still be effective and co-evolve with the new versions. People in different communities can select different versions.

Figure 6 depicts the relationship among Cyber Physical Systems, computing platforms (Grid, P2P, Cloud Computing, etc.), Web x.0, and their co-evolution with sciences and technologies.

Web 4.0 will be a massive knowledge Web, which has the following features:

(1) Massive users who are willing to explain Web resources according to certain incentive mechanisms.

(2) Automatic accumulation of relational knowledge by

- discovering semantic relations between contents, between people, and between people and meta information;

- clustering texts and then constructing content classification hierarchies;

- discovering communities in the semantically linked network and

- carrying out relational reasoning, analogical reasoning and inductive reasoning. 


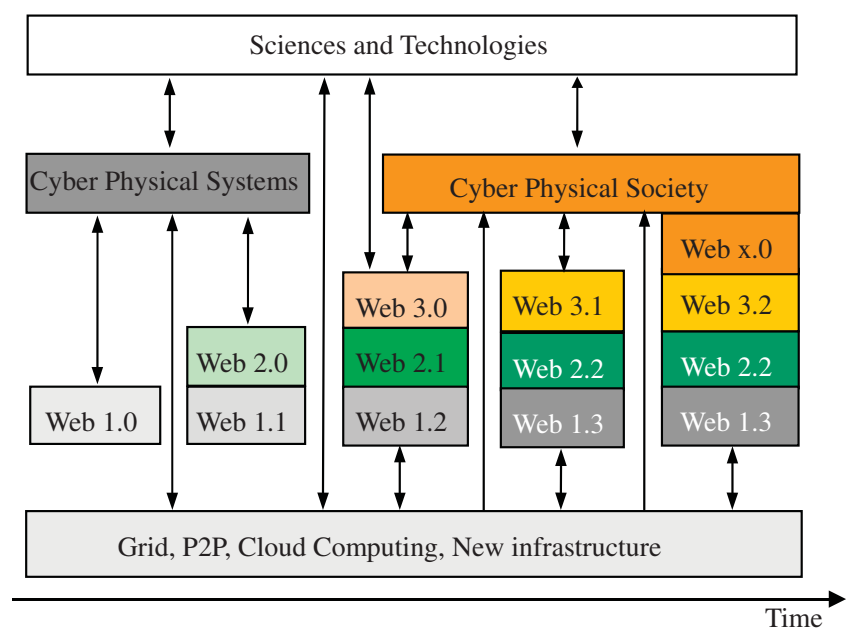

Figure 6. Cyber Physical Society and other systems.

(3) Accumulating problem-solving knowledge by various question-answering mechanisms.

(4) Decentralized relational query and reasoning over semantically linked contents.

(5) Mechanisms of using relational knowledge to explain contents, using problem-solving knowledge to explain contents and relational knowledge, and using relational knowledge to complete the problem-solving knowledge.

(6) Automatic evolution of relational knowledge with massive information sharing behaviors.

(7) Query loosely couples with the structure of resource organization.

(8) Architecture for resource organization is scalable and can adapt to change of resources.

Web X.0 will link to the nature via various sensors, and will co-evolve with our culture and society. During evolution, fragmenting the uniform Web architecture and technology into communities will be unavoidable according to the diversity requirement of the Cyber Physical Society. The facture of the Web by no means return to information isolation. It will be a higher-level development spiral. Fragments will become communities connecting each other by semantic links. Hence, the architecture of the Cyber Physical Society will be a decentralized system consisting of communities of various types.

\section{DECENTRALIZED EVOLUTION MODEL}

The natural ecosystem consists of evolving local ecosystems due to the locality of such factors as climate, land and individual activities. In this decentralized system, different species efficiently make use of resources in local ecosystem accompanied with energy flow, material flow and information flow.

Figure 7 depicts the decentralized evolution model of the Cyber Physical Society consisting of local communities. Diverse technologies will co-exist to support different types of communities. Diverse technologies support communities and will evolve with the communities. One community influences the other communities by various flow cycles as well as social and economic policies. Different communities may adopt different social systems. Each community consists of individuals and evolving species that compete and help each other for reputation or interest.

An individual can get what it needs and provides services for others within community. Every individual is not only consumer but also producer. Those intelligent individuals can play the role of decomposer to decompose a resource into pieces and reconstruct them into meaningful resources that may be useful for other individuals. This decentralized architecture supports the diversity of culture and technology. 


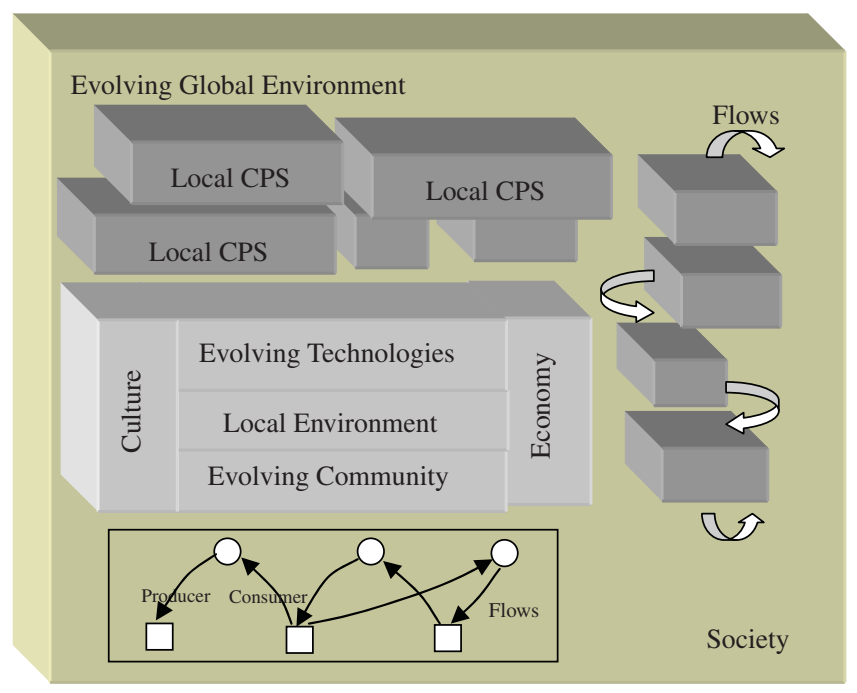

Figure 7. A decentralized evolution model.

\section{BENEFIT OF CPSE RESEARCH}

Carrying out basic research on CPSE can bring the following benefits:

(1) Exploring a new branch of sciences. Exploring CPSE will lead to the fusion of sciences to develop a new science for the development of the future world.

(2) Rethinking existing system and software methodology. Cyber Physical Society is a future existence integrating artificial system and society; hence, research methodology will be different from that of the Web, where large amount of data is available. The problem of CPSE research is that experimental data is not available directly. The methodology will be valuable in science.

(3) Rethinking natural ecology. Research on CPSE will help ecologists rethink their research scope and research objects, and will be a significant reference to research on natural ecology.

(4) Rethinking social science. The Cyber Physical Society will influence human society more directly and substantially than the Web. How will it influence people of different communities? Humans' reading scope is very limited compared with the expanding resources. How to form a healthy local virtual Cyber Physical Society around people is an interesting research issue. Various interactions among individuals in the local Cyber Physical Society provide appropriate personalized services for people. Research on CPSE will help make policies for harmonious development of society, and help make policy for sustainable development of the Web. The development of technologies should be in line with the development of human being in such aspects as knowledge, skill, culture and art.

(5) Rethinking computer science. A global ecosystem consists of local ecosystems. One local ecosystem influences others by material flow and climate. This localization is formed with local characteristics such as climate, land and water. The energy limitation of individuals limits the scale of the food web and also influences the localization of the ecosystem. The computer science is largely based on Turing Machine and algorithms. But Turing Machine is not suitable for all requirements or is not the unique solution. Computer scientists can learn from the natural ecosystem to create a new computing model (e.g. ecological computing) inspired from the intrinsic principles in natural ecosystem.

(6) Rethinking current Web research. CPSE research will form helpful reference to the current research on the Web and Semantic Web. 


\section{ORGANIZING RESOURCES IN THE CYBER PHYSICAL SOCIETY}

\subsection{Cyber-physical-social semantic link network (CPS-SLN)}

Link is one of the most basic means to organize resources. Various explicit and implicit links in the Cyber Physical Society will constitute a self-organized cyber-physical-socio semantic link network(CPS-SLN) $[10,12,16]$. Semantic links connect nodes in spaces to transform interactions. Rules in different spaces follow different rules.

The semantics of semantic nodes and semantic links are specified by concept hierarchy and rules. The first-level of the concept hierarchy regulates commonsense, and the second-level regulates domain commonsense. Users can use their own keywords to tag semantic nodes and semantic links by extending the hierarchy. The frequently used tags can be regarded as commonsense by linking them to the existing classes.

The importance of a semantic link is in positive proportion to: the importance of its two ending nodes, its occurrence in the network the times of interactions through it, and the times it participates in semantic reasoning. The importance of a semantic node is in positive proportion to the importance of its neighbor nodes. The probability of a semantic link is in positive proportion to the probability of its premise links if there is a reasoning rule. The probability of a node is determined by the probability it belongs to a category.

CPS-SLN has the following characteristics: through spaces; self-organized, relational reasoning; and emerging semantics. Structural communities and semantic communities will be formed in CPSSLN due to its semantic self-organization feature. Different from previous graph-based community discovery, semantic communities are specific to semantics and reasoning rules.

\subsection{Cyber-physical-socio resource space model (CPS-RSM)}

Classification is one of the most basic ways to recognize the real world and manage resources in physical space. Bookshelf, drawer, and folder are tools for organizing resources in physical space. The cyber-physical-socio resource space model (CPS-RSM) is a semantic space model for specifying, storing, managing and locating the contents of resources by normalizing classifications [28]. An $n$-dimensional CPS resource space represents $n$ kinds of classification method. Selecting one coordinate at every dimension can uniquely locate a point-a category of resources.

A CPS resource space is a multi-dimensional classification space where dimensions are discrete and every coordinate can be a coordinate tree, where low-level coordinates finely classify their parent coordinates. The intrinsic characteristics of the CPS-RSM determine its research value since it is not a distance space with linear dimensions. A point in the space can be a set of resources, a set of semantic links or a resource space. A resource space can be normalized to ensure the correctness of managing resources by setting constraints on dimensions [12, 28].

CPS-SLN represents self-organization and the CPS-RSM represents normalization of organizing resources. The semantic links can establish ties between resources and between spaces. The CPSRSM can be used to normalize the classification trees. The integration of the CPS-SLN, CPS-RSM and other models can provide a stronger semantic model for organizing resources in the Cyber Physical Society [30, 31].

The Cyber Physical Society needs a scalable, autonomous, adaptable, automated, normalized, self-managed, self-organized, decentralized, evolving and dynamic scalable Platform [9]. Various semantic P2P overlays are candidate techniques [32-35].

\subsection{Cyber-physical-socio resource planning (CPSRP)}

Enterprise Resource Planning (ERP) is to facilitate information flow between business functions within organization and manage connections to outside stakeholders. Cyber-physical-socio resource planning(CPSRP) has the following distinguished characteristics:

(1) Information flow will be extended to service flow, knowledge flow, value flow and material flow as well as the coordination of different types of flows. Flow planning concerns not only intra-enterprise but also inter-enterprise harmony. 
(2) Resource planning will emphasize more on global or regional development than single enterprise, with the help of cyber space. Enterprise planning will be carried out under the restriction of global or regional development.

(3) Sustainable economical, ecological and social development is the goal.

(4) Enterprises will know real requirements, raw materials, production capacities, markets and emissions in real-time through various sensors. Warehouse cost will be minimized. Emission will be controlled by decomposing the waste and exchanging emission right in the cyber physical market.

(5) Micro production and business processes will dynamically link to macro economic, social and natural effects in real-time through the cyber space so that foreseeable measures can be taken.

(6) Realizing knowledge and innovation management in mental space. Knowledge is the most precious property of enterprises. Innovation is the original force of enterprise development. The effect of management will be reflected in real-time; hence, adaptive measures can be taken on time.

(7) Recommending suitable upstream or downstream partners to enterprise to extend flow networks. It is ideal to form recycle flow networks so that waste can be used as possible.

\section{CONCLUSION}

The Cyber Physical Society will be a Cyber Physical Socio ecosystem, where natural physical space, socio space, mental space and cyber space harmoniously interact with each other and coevolve [8]. It will accelerate the development of sciences, technologies, economy and culture. Artificial intelligence concerns human wisdom and intelligent machines. CPSE concerns the relationship between individuals in the cyber-physical-socia environment, between individuals and communities, and between humans and environment. CPSE will be a multi-disciplinary science on research, planning, development and evolution of the future Cyber Physical Society.

\section{ACKNOWLEDGEMENTS}

This work was supported by International Cooperation Project of Ministry of Science and Technology of China (2006DFA11970), National High Technology Research and Development Program of China (2007AA12Z220), National Science Foundation of China (60773057 and 60703018), and National Science Foundation of China (Grant no.70773109).

\section{REFERENCES}

1. Berners-Lee T, Hendler J, Lassila O. The semantic web. Scientific American, 2001.

2. Berners-Lee T, Hall W, Hendler JA, O'Hara K, Shadbolt N, Weitzner DJ. A framework for web science. Foundations and Trends in Web Science 2006; 1-130.

3. De Roure D, Jennings NR, Shadbolt NR. The semantic grid: Past, present, and future. Proceedings of the IEEE 2005; 93(3):669-681.

4. Foster I. Internet computing and the emerging grid. Nature 2000.

5. Foster I. Service-oriented science. Science 2005; 308(5723):814-817.

6. Hey T, Trefethen AE. Cyberinfrastructure for e-science. Science 2005; 308(5723):817-821.

7. Zhuge H. Semantic grid: Scientific issues, infrastructure, and methodology. Communications of the ACM 2005; 48(4):117-119.

8. Zhuge H, Shi X. Toward the eco-grid: A harmoniously evolved interconnection environment. Communications of the ACM 2004; 47(9):78-83.

9. Zhuge H. The future interconnection environment. IEEE Computer 2005; 38(4):27-33.

10. Zhuge H. Socio-natural thought semantic link network: A method of semantic networking in the cyber physical society. The 24th International Conference on Advanced Information Networking and Applications, IEEE AINA2010 (Keynote), Perth, Australia, April 2010; 19-26.

11. Zhuge H. China's e-science knowledge grid environment. IEEE Intelligent Systems 2004; 19(1):13-17.

12. Zhuge H. The Knowledge Grid. World Scientific: Singapore, 2004.

13. Girvan M, Newman M. Community structure in social and biological networks. Proceedings of National Academy of Sciences of the United States of America, vol. 99(12), 2002; 8271-8276. 
14. Wilkinson DM, Huberman BA. A method for finding communities of related genes. Proceedings of National Academy of Sciences of the United States of America 2004; 101:5241-5248.

15. Zhuge H. Discovery of knowledge flow in science. Communications of the ACM 2006; 49(5):101-107.

16. Zhuge H. Interactive semantics. Artificial Intelligence 2010; 174:190-204.

17. Bronfenbrenner U. The Ecology of Human Development: Experiments by Nature and Design. Harvard University: Harvard, 1979.

18. Danchin É, Giraldeau L, Valone TJ, Wagner RH. Public information: From nosy neighbors to cultural. Science 2004; 305(23):487-491.

19. Kleinberg J. Navigation in a small world. Nature 2000; 406:845.

20. Nowak MA, Sigmund K. Evolution of indirect reciprocity. Nature 2005; 427(27):1291-1298.

21. Bush V. As we may think. Atlantic Monthly, July 1945.

22. Booch G. Object Oriented Design with Applications. Benjamin/Cummings: Redwood City, CA, 1991.

23. Ferber J. Multi-agent Systems: An Introduction to Distributed Artificial Intelligence. Addison Wesley Longman: Harlow, 1999.

24. Gray J. What next?: A dozen information-technology research goals. Journal of ACM 2003; 50(1):41-57.

25. Zhuge H. The knowledge grid environment. IEEE Intelligent Systems 2008.

26. Shannon CE, Weiner W. The Mathematical Theory of Communication. University of Illinois Press: Urbana, IL, 1963.

27. Zhuge H. The Web Resource Space Model. Springer: Berlin, 2007.

28. Zhuge H, Li X. Peer-to-peer in metric space and semantic space. IEEE Transactions on Knowledge and Data Engineering 2007; 6(19):759-771.

29. Barabási AL, Albert R. Emergence of scaling in random networks. Science 1999; 286:509-512.

30. Codd EF. A relational model of data for large shared data banks. Communications of the ACM 1970; 13(6):377-387.

31. Zhuge H. Communities and emerging semantics in semantic link network: Discovery and learning. IEEE Transactions on Knowledge and Data Engineering 2009; 21(6):785-799.

32. Stoica I, Morris R, Karger DR, Kaashoek MF, Balakrishnan H. Chord: A scalable peer-to-peer lookup service for internet applications. Proceedings of the ACM SIGCOMM, CA, U.S.A., August 2001; 149-160.

33. Zhuge H. Chen X, Sun X, Yao E. HRing: A structured P2P overlay based on harmonic series. IEEE Transactions on Parallel and Distributed Systems 2008; 19(2):145-158.

34. Zhuge H, Feng L. Distributed suffix tree overlay for peer-to-peer search. IEEE Transactions on Knowledge and Data Engineering 2008; 20(2):276-285.

35. Zhuge H, Xing Y, Shi P. Resource space model, OWL and database: Mapping and integration. ACM Transactions on Internet Technology 2008; 8(4):1-31. 